This article was downloaded by: [B-on Consortium - 2007]

On: 7 November 2008

Access details: Access Details: [subscription number 778384750]

Publisher Informa Healthcare

Informa Ltd Registered in England and Wales Registered Number: 1072954 Registered office: Mortimer House, 37-41 Mortimer Street, London W1T 3JH, UK

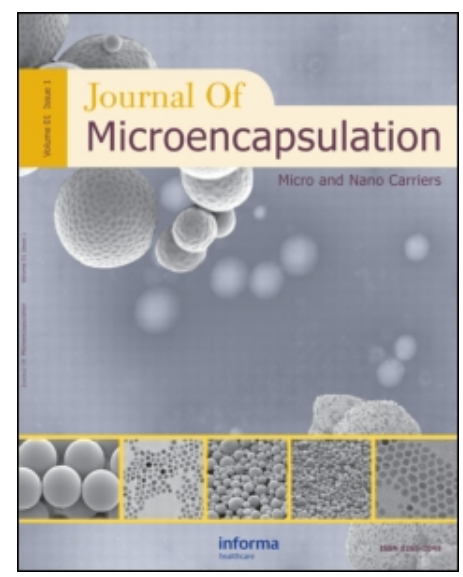

\title{
Journal of Microencapsulation
}

Publication details, including instructions for authors and subscription information:

http://www.informaworld.com/smpp/title content=t713723933

\section{Preparation and characterization of flurbiprofen-loaded poly(3-hydroxybutyrate- co-3-hydroxyvalerate) microspheres}

Patrícia A. Coimbra a; Hermínio C. De Sousa a; Maria H. Gil a

a Departamento de Engenharia Química, Universidade de Coimbra, 3030-790 Coimbra, Portugal

First Published on: 22 January 2008

To cite this Article Coimbra, Patrícia A., De Sousa, Hermínio C. and Gil, Maria H.(2008)'Preparation and characterization of flurbiprofen-loaded poly(3-hydroxybutyrate-co-3-hydroxyvalerate) microspheres',Journal of Microencapsulation,25:3,170 - 178

To link to this Article: DOI: $10.1080 / 02652040701814140$

URL: http://dx.doi.org/10.1080/02652040701814140

\section{PLEASE SCROLL DOWN FOR ARTICLE}

Full terms and conditions of use: http://www.informaworld.com/terms-and-conditions-of-access.pdf

This article may be used for research, teaching and private study purposes. Any substantial or systematic reproduction, re-distribution, re-selling, loan or sub-licensing, systematic supply or distribution in any form to anyone is expressly forbidden.

The publisher does not give any warranty express or implied or make any representation that the contents will be complete or accurate or up to date. The accuracy of any instructions, formulae and drug doses should be independently verified with primary sources. The publisher shall not be liable for any loss, actions, claims, proceedings, demand or costs or damages whatsoever or howsoever caused arising directly or indirectly in connection with or arising out of the use of this material. 


\title{
Preparation and characterization of flurbiprofen-loaded poly(3-hydroxybutyrate-co-3-hydroxyvalerate) microspheres
}

\author{
PATRÍCIA A. COIMBRA, HERMÍNIO C. DE SOUSA, \& MARIA H. GIL \\ Departamento de Engenharia Química, Universidade de Coimbra, Pólo II, Pinhal de Marrocos, \\ Rua Sílvio Lima, 3030-790 Coimbra, Portugal
}

(Received 12 April 2007; accepted 16 November 2007)

\begin{abstract}
Poly(3-hydroxybutyrate-co-3-hydroxyvalerate) (PHBV) microspheres containing flurbiprofen were prepared by an oil-in-water emulsion solvent evaporation method, in order to develop a particulate drug delivery system for localized administration. A response surface method (RSM) using a central composite design was employed to evaluate the effect of the poly(vinyl alcohol) (PVA) $(\%, w / v)$ concentration in the aqueous phase and the PHBV concentration in the organic phase $(\%$, w/v) on some of the resulting microspheres properties. The response variables were the encapsulation efficiency (EE), the mean particle size, the width of particle size distribution (expressed by the SPAN value) and the required time for the in vitro release of $50 \%$ of the encapsulated drug $\left(t_{50 \%}\right)$. Second-order polynomial and linear equations were fitted to experimental data and were also used to interpret the results. Results indicated that the concentration of the stabilizer (PVA) showed a highly negative effect on the EE probably due to the increased drug solubility in the aqueous phase as a result of the higher PVA concentrations. Particle diameter mean size increased with the increased polymer concentration while the width of the particle size distribution was found to decrease with the increase of the stabilizer agent. Finally, results indicated that none of the investigated variables presented a significant effect on the $t_{50 \%}$ values.
\end{abstract}

Keywords: Poly(3-hydroxybutyrate-co-3-hydroxyvalerate), flurbiprofen, microspheres, central composite design

\section{Introduction}

The preparation of microcapsules and microspheres of biodegradable polymers as vehicles for the controlled release of bioactive agents (i.e. drugs, proteins, peptides, genetic material) has been the focus of much investigation and has been a growing research field in recent years (Freiberg and Zhu 2004). Usually, the main goal in the design of a polymeric nano/microparticulated system for delivering a specific drug is to achieve a controlled release rate of the drug into the desired specific site of action and at therapeutic optimal levels. This can be done by tailoring several nano/ microparticles properties such as particle size and particle size distribution, bulk and surface morphology, drug loading capacity and drug load homogeneity, kinetics of drug release, etc. These characteristics will always be defined by the physical and chemical properties of all involved species (polymers, drugs, solvents, surfactants), by the method employed to prepare the loaded particles and by the several operational parameters of the manufacture process.

Among the large number of polymers and copolymers tested for the preparation of biodegradable microspheres, poly(lactic acid) (PLA) and its copolymer poly(lactic acid-co-glycolic acid) (PLGA) are the most extensively investigated due, essentially, to their excellent biocompatibility and biodegradability properties (Hyon 2000). More recently, polyhydroxyalkanoates (PHAs), a class of biodegradable and biocompatible polyesters of biological origin, started receiving considerable attention as a biomaterial presenting great potential for biomedical and pharmaceutical applications (Pouton and Akhtar 1996). The most common representatives of these type of biomaterials are those usually containing hydroxyalkanoic acid monomeric units, like poly(3-hydroxybutyric acid),

Correspondence: Patrícia Coimbra, Departamento de Engenharia Química Universidade de Coimbra Pólo II, Pinhal de Marrocos Rua, Sílvio Lima, 3030-790 Coimbra, Portugal. Fax: +351 239798 703. E-mail: patrici3@eq.uc.pt 
PHB, and also a related copolymer, poly(3-hydroxybutyrate-co-3-hydroxyvalerate), PHBV. Their use as tissue scaffolds and as drug delivery vehicles have been reported (Pouton and Akhtar 1996, Williams et al. 1999, Khang et al. 2001, Baran et al. 2002, Chen and Davis 2002). Their in vivo performances are also being currently investigated (Qu et al. 2006).

The oil-in-water $(\mathrm{O} / \mathrm{W})$ emulsion solvent evaporation method is a common procedure to microencapsulate water-insoluble drugs within water-insoluble polymers (O'Donnell and McGinity 1997, Freitas et al. 2005). Usually, this process involves the dissolution of a hydrophobic drug in a solution of a volatile organic solvent and a water-insoluble polymer. Then, the emulsification of the polymer + drug containing organic phase is done within an aqueous phase and the organic solvent is removed (using heat, freeze-drying or vacuum), resulting in the precipitation of the polymeric material into drug-loaded microspheres. Several process variables will influence the final characteristics of the microspheres prepared by this method, like the aqueous solubility of the drug, the type and amount of used surfactant, the nature of the oil/organic phase, the oil-to-water phase ratio, the drug-to-polymer phase ratio, the stirring speed and the operational temperature (O’Donnell and McGinity 1997).

The objective of this work is to make and characterize drug-loaded PHBV microsphere prepared by the oil-in-water $(\mathrm{O} / \mathrm{W})$ emulsion solvent evaporation method. To evaluate the influence of some process variables in final physical-chemical properties of the obtained microspheres, a response surface experimental design was planned and implemented. Two variables were investigated: the concentration in the aqueous phase of the emulsion stabilizer, poly(vinyl alcohol) (PVA), and the PHBV concentration in the organic phase. The experimental design, classified as a surface response central composite design (Montgomery 2001), was chosen since it allows the understanding of the influence of the above referred two combined factors on the microspheres properties with a minimum of experimental assays. In particular, it is possible to estimate second-order polynomial equations and to map the response over the experimental domain in order to determine optimum conditions for the formulations.

Flurbiprofen was chosen as the model drug to encapsulate. This compound is a potent non-steroidal anti-inflammatory drug (NSAID) used in the treatment and control of the signs and symptoms of several inflammatory chronic processes, like rheumatoid arthritis ( $\mathrm{Lu}$ et al. 2007) and periodontal diseases (Samati et al. 2006). It is also commonly used in ophthalmology to reduce and prevent post-operative inflammations (Vega et al. 2006, Pignatello et al. 2002). Like other NSAIDs, the oral administration of this drug is limited because of the documented toxicity at the gastrointestinal level (Cryer and Feldman 1998).
To prepare more safe and efficient formulations of this drug, several authors have encapsulated it in biodegradable microspheres and nanospheres, intended as possible localized delivery systems. Examples are the work of Samati et al. (2006) that investigated the encapsulation of the salt form of flubiprofen in copolymers of PLGA as a drug delivery system for the periodontal pocked. Vega et al. (2006) also investigated the encapsulation of flurbiprofen in PLGA nanospheres as a possible ocular drug delivery system, while $\mathrm{Lu}$ et al. (2007) recently prepared flurbiprofen gelatine microspheres for intra-articular administration in the joint cavity.

\section{Experimental}

\section{Experimental design}

A central composite experimental design was used to study the effects of two formulation factors on some of the microspheres properties. The two investigated factors (independent variables) were the stabilizer agent concentration in the aqueous phase $(\% \mathrm{w} / \mathrm{v}$ PVA) and the polymer concentration in the organic phase $(\% \mathrm{w} / \mathrm{v}$ PHBV).

The analysed dependent variables were the encapsulation efficiency (EE), the mean particle size, the width of particle size distribution (expressed by the SPAN value) and the required time for the in vitro release of $50 \%$ of the encapsulated drug $\left(t_{50 \%}\right)$. All the remaining factors, such as the oil-to-water phase ratio, drugto-polymer ratio, stirring speed and temperature, were kept constant. Each independent variable was studied at five levels, with an $\alpha$-value of 1.414 and three replicates for the centre point. Table I shows the coded levels and actual values of the investigated factors. Design-Expert $^{\circledR} \quad($ Version 6.0.10, Stat-Ease Inc, Minneapolis, USA) was used for the generation of the experimental design, modelling and statistical evaluation.

\section{Materials}

Poly(hydroxybutyrate-co-hydroxyvalerate), PHBV (6\% mol HV, Mw $=223 \mathrm{kDa}$ ), was purchased from PHB Industrial SA (Brazil). Flurbiprofen (purity $>98 \%$ ) and poly(vinyl alcohol), PVA ( $80 \%$ hydrolysed, av. Mw $13-23 \mathrm{kDa}$ ), were supplied by Sigma-Aldrich

Table I. Coded and actual values of the investigated factors.

\begin{tabular}{lccccc}
\hline & \multicolumn{5}{c}{ Levels } \\
\cline { 2 - 6 } Factors & -1.414 & -1 & 0 & 1 & 1.414 \\
\hline$X_{1}:$ PVA concentration $(\%, \mathrm{w} / \mathrm{v})$ & 0.38 & 1.00 & 2.50 & 4.00 & 4.60 \\
$X_{2}:$ PHBV concentration $(\%, \mathrm{w} / \mathrm{v})$ & 0.88 & 1.50 & 3.00 & 4.50 & 5.12 \\
\hline
\end{tabular}


(St. Louis, USA). Chloroform was obtained from José A. Vaz Pereira (Lisbon, Portugal).

\section{Preparation of microspheres}

Flurbiprofen-containing PHBV microspheres were prepared by the traditional oil-in water $(\mathrm{O} / \mathrm{W})$ emulsion solvent evaporation technique. To a solution of PHBV in chloroform (used compositions: 0.88, 1.50, 3.00, 4.50 and $5.12 \%, \mathrm{~g} \mathrm{~mL}^{-1}$ ), was added and dissolved a determined amount of flurbiprofen (3 $\mathrm{g}$ flurbiprofen/ $10 \mathrm{~g}$ PHBV). After, $10 \mathrm{~mL}$ of the organic solution was dropped slowly into a mechanical stirred glass reactor, charged with $100 \mathrm{~mL}$ of a PVA solution (used compositions: $0.38,1.00,2.50,4.00$ and $4.60 \%$, $\mathrm{g} \mathrm{mL}^{-1}$ ) to form the $\mathrm{O} / \mathrm{W}$ emulsion. The reactor was immersed in a water-bath at a constant and controlled temperature $\left(30^{\circ} \mathrm{C}\right)$. The obtained $\mathrm{O} / \mathrm{W}$ emulsion was continuously stirred, at $1400 \mathrm{rpm}$ and for $4 \mathrm{~h}$, to permit the evaporation of the organic solvent. The resulting microspheres were collected by centrifugation, at $3000 \mathrm{rpm}$, and then washed three times with deionised water and dried in a vacuum oven (at $40^{\circ} \mathrm{C}$ ). The dried microspheres were stored in a dessicator until further use.

\section{Experimental drug loading and encapsulation efficiency}

The microspheres drug content was determined by UV spectrophotometry. A weighed amount $(\sim 10 \mathrm{mg})$ of drug-loaded PHBV microspheres was dissolved in $1 \mathrm{~mL}$ of chloroform, followed by the addition of $9 \mathrm{~mL}$ of methanol to precipitate the polymer. The resulting suspension was centrifuged and the supernatant was filtrated and analysed in a UV spectrophotometer (Jasco, model V-550) at $247 \mathrm{~nm}$. A calibration curve was generated, at the same wavelength, using pre-prepared standard solutions of flurbiprofen in chloroform/methanol mixtures (1:9). Triplicate measures were performed for each batch. Experimental drug loading was calculated as follows:

$$
\begin{aligned}
& \text { Experimental drug loading } \\
& =\frac{\text { weight of detected drug }}{\text { weight of drug loaded microspheres }}
\end{aligned}
$$

Theoretical drug loading was kept constant to all batches $(23 \%)$ and was defined as:

\section{Theoretical drug loading}

$$
=\frac{\text { weight of added drug }}{\text { weight of added drug }+ \text { weight of added polymer }}
$$

Encapsulation efficiency (EE, \%) was expressed as the ratio between the experimental drug and the theoretical drug loadings.
Determination of flurbiprofen solubility in PVA aqueous solutions

The solubility of flurbiprofen in $0.50 \%(\mathrm{w} / \mathrm{v})$ and $4.50 \%$ PVA aqueous solutions was measured at $25^{\circ} \mathrm{C}$. A known amount of flurbiprofen was added to $20 \mathrm{~mL}$ of PVA solutions, in closed flasks, and left to dissolve during 5 days under magnetic stirring. Aliquots of the saturated solutions were then drawn, conveniently diluted and analysed for absorbance intensity in a UV spectrophotometer (Jasco, model V-550) at $247 \mathrm{~nm}$.

Flurbiprofen content was calculated using standard calibration curves obtained with aqueous solutions with the same PVA concentrations. Triplicate flurbiprofen saturated PVA solutions were prepared for each concentration.

\section{Particle size distribution}

Microspheres particle size and particle size distribution were measured by a laser light scattering analyser (Coulter, model LS130). A suitable amount of dried microspheres, from each formulation was suspended in deionized water and sonicated before measurement. The resulting homogenized suspension was then analysed for the volume mean diameter and particle size distribution. Frauenhofer's method was used to calculate the particle size distributions. The width of these distributions was expressed in terms of SPAN factor, defined as:

$$
S P A N=\frac{d_{90}-d_{10}}{d_{50}}
$$

In this equation, $d_{10}, d_{50}$ and $d_{90}$ represent the diameter value for which a given percentage of particles is smaller than that size. Therefore, the SPAN value indicates the spread of the particle size distribution and small SPAN values are indicative of narrow particle size distributions.

\section{Surface morphology}

Microspheres particle shape and particle surface morphologies were analysed by scanning electron microscopy (SEM). Microspheres were placed on double-sided graphite tape attached onto a metal surface and coated with copper on a sputtering coater. Observations were performed at $20 \mathrm{kV}$ with a JSM-5310 (JEOL, Japan) scanning electron microscope.

\section{In vitro drug release studies}

A dialysis method was used for the in vitro kinetics of drug release studies. A certain amount of drug-loaded microspheres, containing $\sim 1 \mathrm{mg}$ of flurbiprofen, were weighted and added to dialysis bags (with a cutoff of $12-14 \mathrm{kDa}$ ). Bags were filled with $20 \mathrm{~mL}$ of saline solution $\left(3.5 \mathrm{~g} \mathrm{~L}^{-1} \mathrm{Na}^{+}, 5.4 \mathrm{~g} \mathrm{~L}^{-1} \mathrm{Cl}^{-}, \mathrm{pH} \approx 6.0\right)$ and 
introduced in beaker flasks filled with $50 \mathrm{~mL}$ of saline solution. The release medium was stirred at $120 \mathrm{rpm}$ at a constant temperature $\left(37^{\circ} \mathrm{C}\right)$. Then, $1.6 \mathrm{~mL}$ of solution was drawn out at pre-determined time intervals and the release medium was replenished with the same volume of fresh saline solution. Flurbiprofen content was determined by UV spectrophotometry at $247 \mathrm{~nm}$. For comparison, the dissolution of $\sim 1 \mathrm{mg}$ of free drug and a physical mixture of drug and blank PHBV microspheres was also performed at the same conditions.

\section{Thermal analysis}

Thermal analyses of the microspheres and of the pure drug were performed with a modulated differential scanning calorimeter (TA Instruments, model Q100 MDSC). Samples of $2.5-12 \mathrm{mg}$ were placed into aluminium containers and heated, at a constant rate $\left(5^{\circ} \mathrm{C} \mathrm{min}^{-1}\right)$, from 20 to $200^{\circ} \mathrm{C}$ under a nitrogen atmosphere.

\section{Results and discussion}

The use of a response surface method experimental design permits the construction of second-order polynomial models that can describe quantitatively the linear, quadratic and interaction effects of the selected factors on the studied response variables. For two factors, the general model corresponds to the following equation:

$$
Y=b_{0}+b_{1} X_{1}+b_{2} X_{2}+b_{11} X_{1}^{2}+b_{22} X_{2}^{2}+b_{1} X_{1} X_{2}
$$

In this equation, $X_{1}$ and $X_{2}$ are the independent variables (factors) and $Y$ is the investigated dependent variable (response). Also, $b_{0}$ represents the arithmetic average of all quantitative outcomes of all the runs, $b_{1}$ and $b_{2}$ are related with the independent variables effect on the response, $b_{11}$ and $b_{22}$ are two quadratic relationships and $b_{12}$ represents the interaction effect between the two variables. A coefficient with a positive sign signifies a synergistic effect, whereas a negative sign stands for an antagonistic effect.

As referred, the independent variables studied in this work were the PVA concentration in the aqueous phase $\left(X_{1}\right)$ and the PHBV concentration in the organic phase $\left(X_{2}\right)$, while the analysed response variables were the encapsulation efficiency $\left(Y_{1}\right)$, the mean particle size of the microspheres $\left(Y_{2}\right)$, the width of the particle size distribution, expressed by the SPAN value $\left(Y_{3}\right)$ and the time for the in vitro release of $50 \%$ of the encapsulated drug $\left(Y_{4}\right)$. DesignExpert $^{\circledR}$ software was the employed tool to analyse the response variables and to generate the polynomial models. Model simplification was carried out by eliminating insignificant parameters (those parameters with the higher $p$-values) in the polynomial equations resulting from multiple regression analysis. The obtained models to describe the dependent variables were selected taking in account their statistical significance $(p<0.01)$. The experimental runs of the implemented central composite design and the responses of the investigated dependent variables are listed in Table II.

\section{Encapsulation efficiency}

The values for the flurbiprofen in PHBV microspheres encapsulation efficiency (EE) $\left(Y_{1}\right.$, Table II) ranged between $20.9 \pm 0.7 \%$ (4\% PVA, $1.5 \%$ PHBV) and $71.1 \pm 2.1 \% \quad(0.38 \%$ PVA, $3 \%$ PHBV $)$, which corresponds to experimental drug loadings of $4.8 \pm 0.2 \%$ and $16.4 \pm 0.5 \%$, respectively. The obtained values for this response were fitted by a quadratic model using the least squares method $\left(r^{2}=0.9331\right)$ :

$$
\begin{aligned}
E E(\%)= & 56.6-24.5 P V A+7.9 P H B V+2.4 P V A^{2} \\
& -0.8 P H B V^{2}+1.9 P V A \times P H B V
\end{aligned}
$$

The obtained regression coefficients show that $\mathrm{EE}$ is significantly influenced by the two investigated factors but in opposite ways $(p<0.01)$. In addition, the positive quadratic effect of PVA appears to be

Table II. Experimental runs and responses of the central composite experimental design.

\begin{tabular}{lcccccc}
\hline Batch & $\begin{array}{c}X_{1} \text { PVA } \\
(\%, \mathrm{w} / \mathrm{v})\end{array}$ & $\begin{array}{c}X_{2} \text { PHBV } \\
(\%, \mathrm{w} / \mathrm{v})\end{array}$ & $Y_{1}$ EE $(\%)$ & $\begin{array}{r}Y_{2} \text { Mean } \\
\text { size }(\mu \mathrm{m})\end{array}$ & $Y_{3}$ SPAN & $Y_{4} t_{50 \%}(\mathrm{~min})$ \\
\hline $1^{*}$ & 2.5 & 3 & $40.6 \pm 1.4$ & 8.7 & 2.2 & 105 \\
2 & 0.38 & 3 & $71.1 \pm 2.1$ & 7.0 & 3.7 & 108 \\
3 & 4.62 & 3 & $29.6 \pm 1.9$ & 10.4 & 1.8 & 123 \\
4 & 2.5 & 0.88 & $22.0 \pm 1.0$ & 4.6 & 1.7 & 112 \\
5 & 4 & 1.5 & $20.9 \pm 0.7$ & 5.4 & 2.2 & 105 \\
6 & 1 & 1.5 & $42.5 \pm 1.1$ & 9.9 & 3.5 & 145 \\
7 & 4 & 4.5 & $56.0 \pm 1.3$ & 8.5 & 1.8 & 155 \\
8 & 1 & 4.5 & $60.4 \pm 3.8$ & 11.3 & 4.6 & 220 \\
$9^{*}$ & 2.5 & 3 & $41.9 \pm 1.9$ & 12.0 & 1.6 & 112 \\
10 & 2.5 & 5.12 & $50.2 \pm 0.5$ & 19.2 & 2.0 & 110 \\
$11^{*}$ & 2.5 & 3 & $39.5 \pm 0.7$ & 10.3 & 1.8 & 112 \\
\hline
\end{tabular}

*Centre points. 
statistically significant $(p<0.1)$. On the other hand, the quadratic effect of PHBV and the interaction effect between variables is not statistically different from zero $(p>0.1)$, being kept in the model only to support the hierarchy of the polynomial equation. The relationship between $\mathrm{EE}$ and the independent variables is further illustrated whit a response surface plot, displayed in Figure 1.

The fitted model shows that the stabilizer's concentration in the aqueous phase (\% PVA) is the factor that has a higher impact in the EE, with a large negative effect. In the literature, the reported effect of PVA concentration in the EE of drug-loaded microspheres prepared by the $\mathrm{O} / \mathrm{W}$ solvent evaporation method varies according to the drug/polymer systems investigated. While some authors report an increase of EE with the increase of the stabilizer concentration (Samati et al. 2006) or report that this variable doesn't statistically influence the obtained values of the EE (Bolourtchian et al. 2005), other studies, like the present one, conclude that the increase of the stabilizer concentration has a negative effect in the EE (Lai and Tsiang 2004, Fu et al. 2005).

Considering that, in the solvent evaporation method, the drug entrapment efficiency reflects the partition of the drug between the disperse organic phase and the continuous aqueous phase during microspheres solidification and precipitation, the decrease of EE with the increase in PVA concentration can be explained by the resulting solubility enhancement of flurbiprofen in the aqueous phase due to the presence of these increasing amounts of PVA. In fact, the solubility measurements of flurbiprofen in PVA aqueous solutions showed a significantly solubility increase from

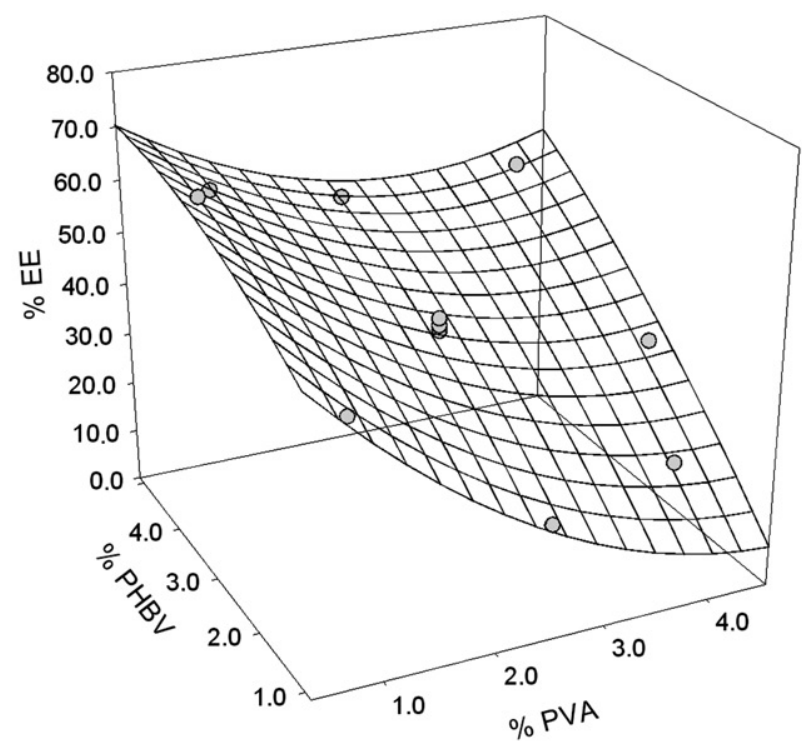

Figure 1. Response surface plot showing the effect of PHBV concentration and PVA concentration on the encapsulation efficiency of the flurbiprofen-loaded microspheres.
$0.173 \pm 0.010 \mathrm{mg} \mathrm{mL}^{-1}$ in a $0.5 \%$ PVA solution, up to $0.336 \pm 0.006 \mathrm{mg} \mathrm{mL}^{-1}$ in a $4.5 \%$ PVA solution. Compared with the solubility of flurbiprofen in pure water, $\sim 0.01 \mathrm{mg} \mathrm{mL}^{-1}$ (Park and Kim 1999), the solubility of flurbiprofen in the presence of PVA is remarkably enhanced (more than 10-times). This phenomenon, the increase of solubility of poorly water-soluble drugs in surfactant solutions (micellization), is well reported in literature ( $\mathrm{Li}$ and Zhao 2003, Yiyun and Jiepin 2005, Park and Choi 2006), being used as a strategy to improve the bioavailability of water insoluble drugs, in particular NSAIDs.

The other studied factor, the polymer concentration in the organic phase (\% PHBV), has a significantly positive contribution to $\mathrm{EE}$. The increase of $\mathrm{EE}$ with the increase of polymer concentration is well established in the literature and is explained by the viscosity increase of the disperse phase, which restricts the drug migration to the continuous phase (Freitas et al. 2005).

\section{Particle size distribution}

For all the experimental batches, unimodal size distributions were obtained, with mean particle sizes ranging from $4.6 \mu \mathrm{m}$ (batch 4) up to $19.2 \mu \mathrm{m}$ (batch 10) and SPAN indexes between 1.6 (batch 9) and 4.6 (batch 8). After model simplification, with the elimination of the quadratic and interaction terms, the obtained values for the mean particle size $\left(Y_{2}\right)$ and for the SPAN value $\left(Y_{3}\right)$ were fitted to linear models, according to the following equations:

Mean particle size $(\mu \mathrm{m})=4.08-0.23 P V A+2.10 P H B V$

$$
S P A N=3.58-0.57 P V A+0.10 P H B V
$$

The statistical analysis of the models indicates that the concentration of the polymer in the organic phase is the only factor that significantly influences the mean particle size $(p<0.05)$, with a positive effect. This increase of particle size with the increase of polymer concentration is well documented in the literature (O'Donnell and McGinity 1997, Freitas et al. 2005) and it can be explained by the increasing viscosity of the organic phase and, as a consequence, this phase will be more difficult to disperse into small droplets. According to the fitted models, the concentration of the stabilizer has a statistical null effect on the mean particle size $(p>0.1)$ but has a significant negative effect in the width of the particle size distribution $(p<0.01)$. The higher SPAN values were observed for the formulations with $0.38 \%$ and $1 \%$ of PVA concentration (batches 2, 6 and 8), which correspond to the lower levels for this factor in the experimental design. The obtained broader size distributions for these formulations are possible due to the 
microspheres aggregation during solvent evaporation, caused by the low stabilizer concentration in the aqueous phase. In fact, some degree of microspheres aggregation was visible in these samples, in contrast to all the other formulations, which yielded completely free powders.

\section{Surface morphology}

Figure 2 shows the SEM micrographs of the surface of PHBV blank microspheres (Figure 2(a)), flurbiprofen-loaded microspheres (Figure 2(b)) and microspheres after the in vitro release of flurbiprofen in a saline solution (Figure 2(c)). Blank and drug-loaded microspheres were spherical in shape with a very rough surface. This morphology is typical of PHB and PHBV microspheres and is attributed to the highly crystalline character of these polymers (Martin et al. 2000). Blank microspheres had a compact structure with some visible surface pores while drug-loaded microspheres presented large surface cavities, probably derived from the collision between the microspheres during hardening. It is also possible to see fine drug particles coated on the surface of the microspheres. After drug release, microspheres presented a 'washed' aspect, with large holes/pores on the surface and the crystalline polymer morphology is again well visible.

\section{Thermal analysis}

Figure 3 reports the thermal behaviour of pure flurbiprofen (Figure 3 (a)), of blank PHBV microspheres (Figure 3(b)), a physical mixture of flurbiprofen and blank PHBV microspheres (Figure 3(c)) and flurbiprofen-loaded PHBV microspheres (Figure 3(d)). Figure 3(a) displays a narrow endothermic peak at $115.4^{\circ} \mathrm{C}$, corresponding to the solid melting point. Figure 3(b) shows two endothermic peaks of fusion $\left(\sim 145^{\circ} \mathrm{C}\right.$ and $\left.164^{\circ} \mathrm{C}\right)$ for the PHBV blank microspheres. Double and multiple melting behaviour of PHB and PHBV films obtained by solution casting have been reported in the literature (Gunaratne and Shanks 2005) and are attributed to the phenomenon of melting, recrystallization and remelting during heating. Figure 3(c) corresponds to the DSC profile obtained from a physical mixture of flurbiprofen and blank microspheres $(25 / 75, \% \mathrm{w} / \mathrm{w})$ and combines the thermal events previously described. Figure 3(d) illustrates the thermal behaviour of flurbiprofen-loaded-PHBV microspheres with $9.4 \%$ of experimental drug loading (batch 1). The characteristic endothermic melting peaks of the polymer are present but the endothermic melting peak of flurbiprofen is not visible. Similar results were also obtained for DSC studies of other drug-loading polymeric particles prepared by solvent evaporation methods (Freiberg and Zhu 2004). According to Dubernet (1995), the disappearance of the endothermic peak, correspondent to the encapsulated drug melting point, indicates that the drug is dissolved in the polymer as a solid solution or that the drug is dispersed in the polymer in a metastable molecular dispersion. This result is an indication that the flurbiprofen particles that seem to coat the surface of the drug-loaded microspheres in SEM micrographs (Figure 2(b)) are probably in an amorphous state, finely dispersed at the microspheres surface.

\section{In vitro drug release studies}

The flurbiprofen release profiles from PHBV microspheres, in a saline solution of $\mathrm{pH} \approx 6$ and at $37^{\circ} \mathrm{C}$, were found to be very similar for all the prepared batches, showing an initial burst release (of 15-25\% of total drug) in the first half hour and, after $8 \mathrm{~h}$, more than $90 \%$ of total drug was released. The time required to release $50 \%$ of the encapsulated drug $\left(t_{50 \%}\right)$ ranged between $105-155 \mathrm{~min}$ for all the formulations, with the exception of formulation 8, which was $220 \mathrm{~min}$. Data analysis of $t_{50 \%}$ values (Table II) showed that none of the investigated factors had a significant effect on this variable $(p<0.01)$ and formulation 8 was identified as an outlier by the implemented statistical analysis. This means that the fitted model is a constant, corresponding to the mean value of $t_{50 \%}$ of all batches $(128 \mathrm{~min})$. On the other hand, the dissolution profile of the pure drug, alone or as a physical mixture with blank microspheres, evidences a slower release, with a $t_{50 \%}(255 \mathrm{~min})$ significantly $(p<0.1)$ higher than the mean $t_{50 \%}$ for the flurbiprofen-loaded PHBV microspheres. This is illustrated in Figure 4, where the in vitro release profiles from the drugloaded microspheres corresponding to the three central points of the experimental design $(2.5 \%$ PVA, 3\% PHBV) are compared with the dissolution profile of the pure drug and of a physical mixture of flurbiprofen and blank PHBV microspheres (for clarity, the release profiles of the remaining formulations of the experimental design are not represented). In Figure 4 is also clear that the percentage of released drug in the first $30 \mathrm{~min}$ from drug loaded microspheres is higher than the percentage of the pure drug dissolved in the release medium in the same time interval $(\sim 10 \%)$. These results indicate that the drug release from microspheres is essentially controlled by the drug dissolution rate rather than by the hydrolytic degradation of polymeric chains (as expected due to the known slow degradation of PHB and PHBV) or by the diffusion of the drug throughout the polymeric matrix that, according to the SEM images, is a irregular and porous structure, where the entrapped drug can easily leach out by the dissolution medium. The dissolution of poorly soluble water drugs, like flurbiprofen, is affected by several physical properties, including 
(a)
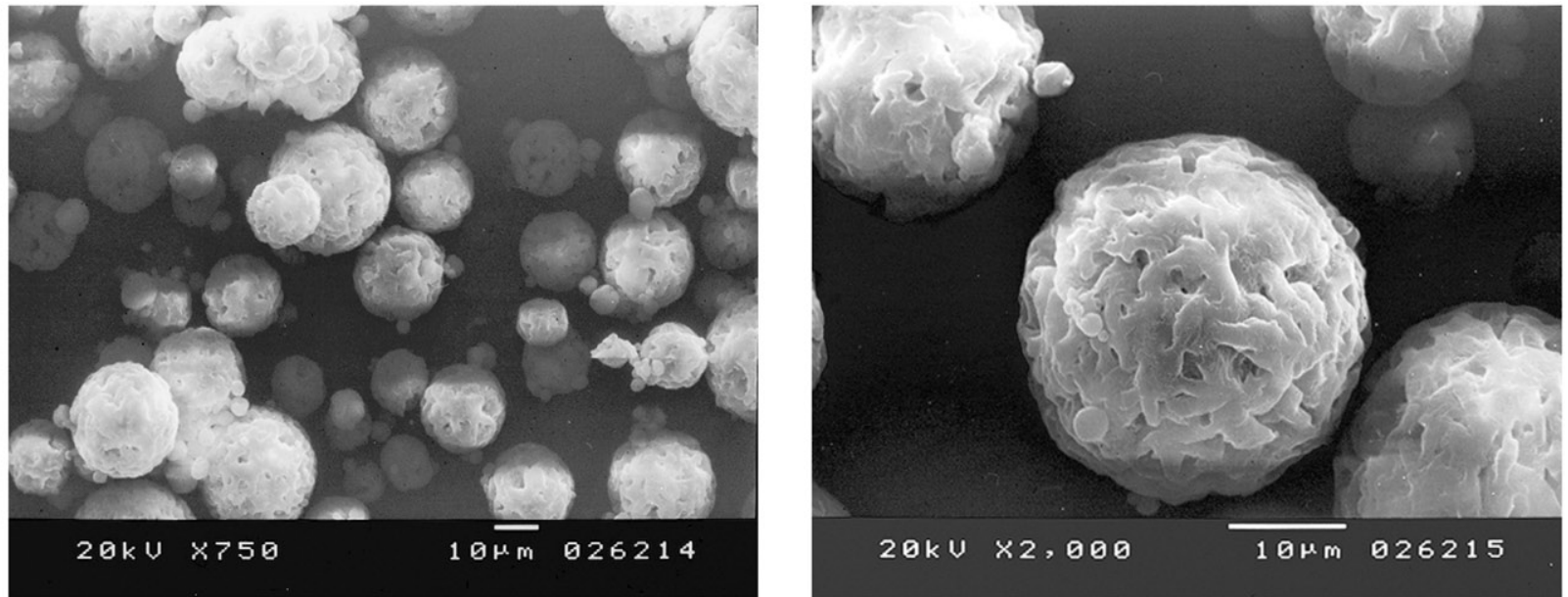

(b)
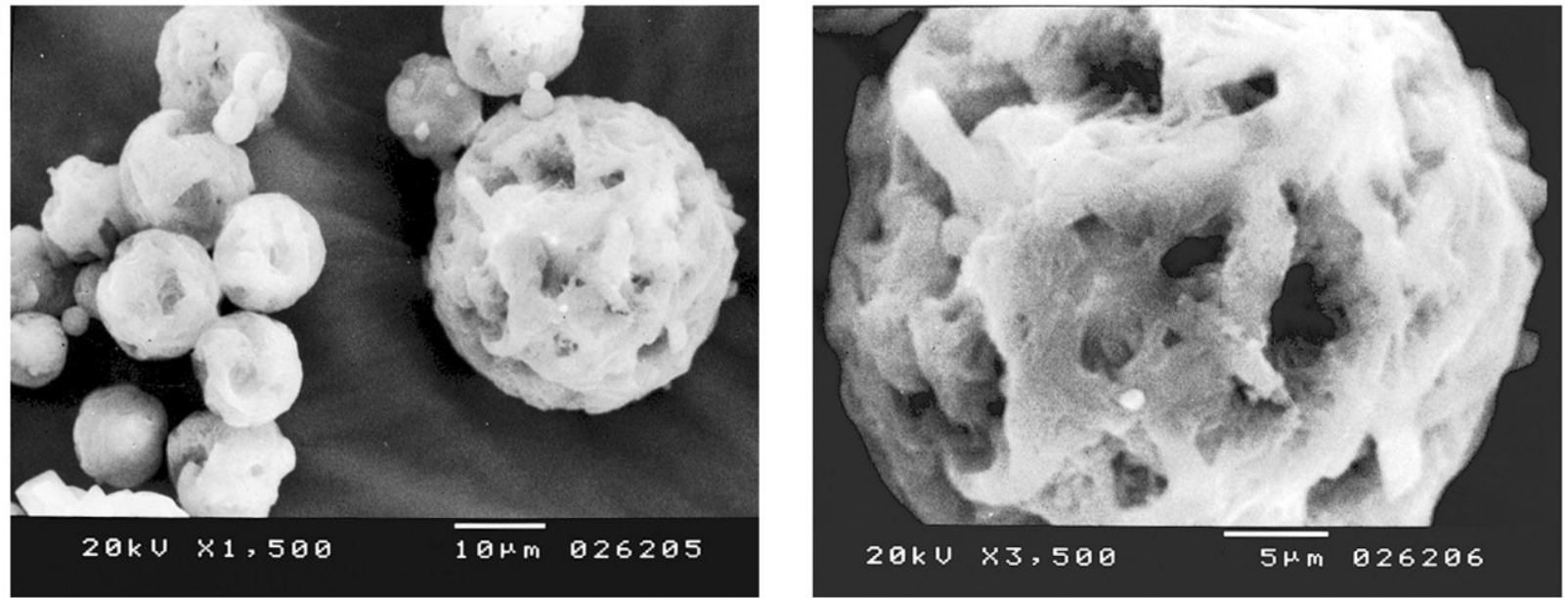

(c)
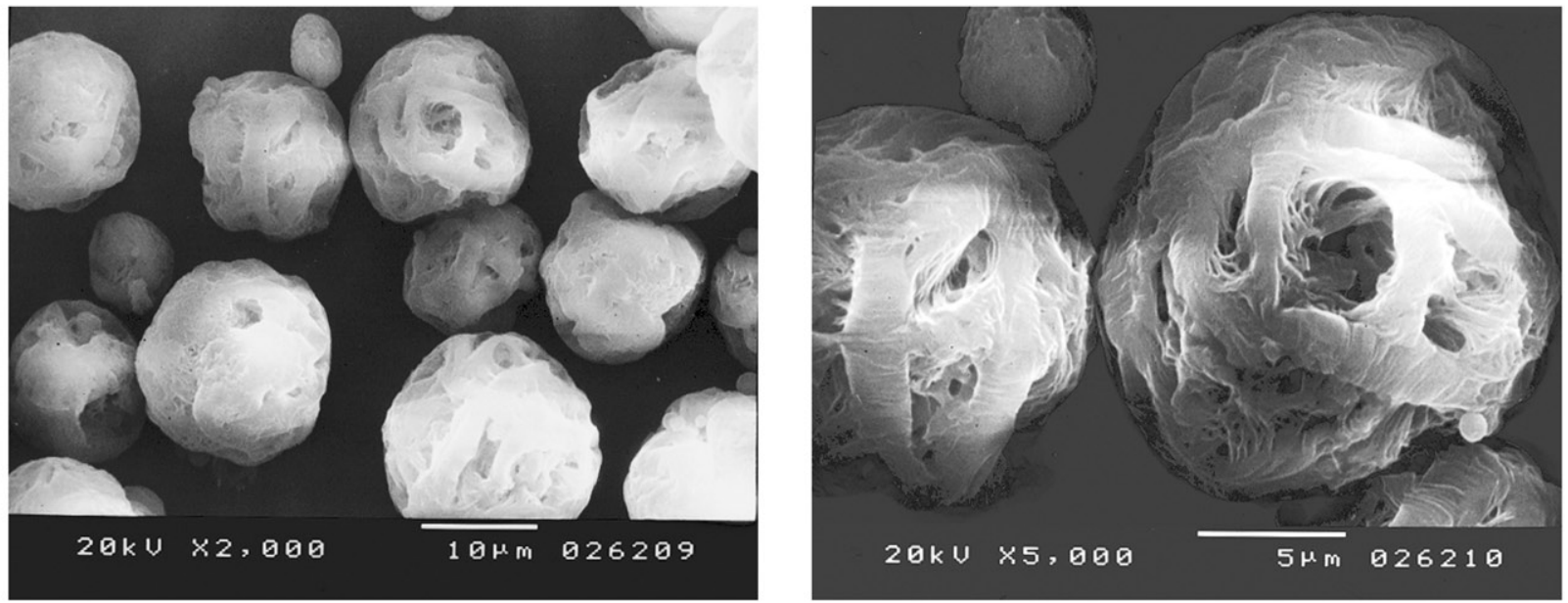

Figure 2. SEM micrographs of: (a) blank microspheres; (b) flurbiprofen-loaded microspheres; (c) microspheres after drug release.

crystal habit, particle size, polymorphism, surface area and wettability (Zaghloul et al. 2001). In this case, the higher dissolution rate of flurbiprofen from PHBV microspheres, when compared to the dissolution rate profile of the pure drug, can be attributed to the dispersion of the amorphous drug in the porous matrix and surface of the microspheres, which enhanced the contact with the dissolution medium, leading to a faster dissolution.

Considering that the drug-to-polymer ratio was kept constant in all formulations, in a ratio of $3: 10$, and that this factor influences the in vitro drug release profile of drug-loaded microspheres prepared by the emulsification solvent evaporation method 


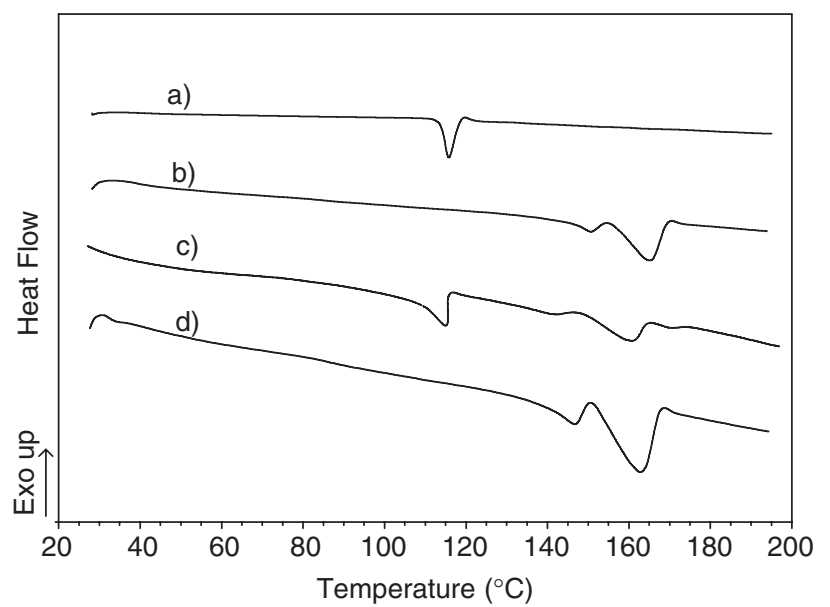

Figure 3. DSC profiles of: (a) flurbiprofen $(2.5 \mathrm{mg})$; (b) blank PHBV microspheres (4.5 mg); (c) physical mixture (3:1) of blank PHBV microspheres and flurbiprofen $(8.1 \mathrm{mg})$; (d) flurbiprofen-loaded PHBV microspheres, batch 1 (12.0 mg).

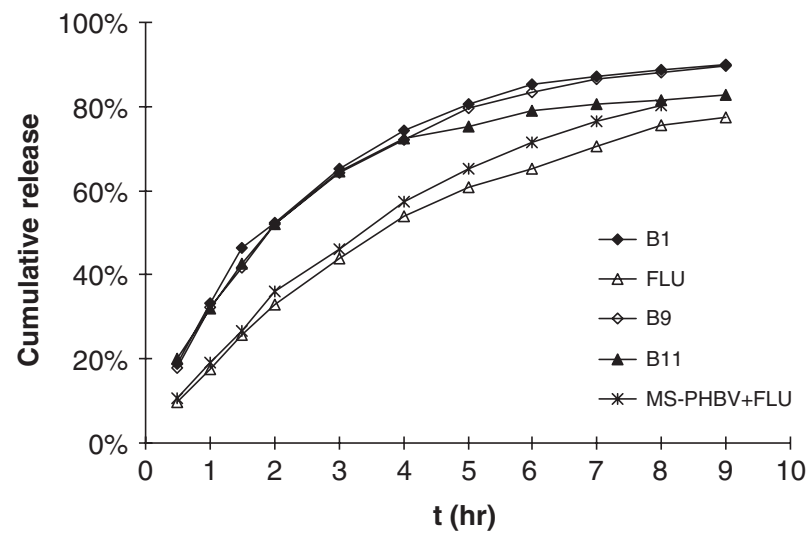

Figure 4. In vitro release profiles of flurbiprofen from PHBV microspheres (batches 1, 9 and 11) and dissolution rate profile of flurbiprofen as pure drug (FLU) and as a physical mixture (1:10) of pure flurbiprofen and blank PHBV microspheres (PHBV + FLU).

(Freiberg and Zhu 2004), in future studies the influence of this factor will be investigated, with the objective of obtaining flurbiprofen-PHBV loaded microspheres with a more prolonged and sustained release profile.

\section{Conclusions}

In order to develop a particulate drug delivery system using biodegradable and biocompatible polyesters of biological origin, poly(3-hydroxybutyrate-co-3hydroxyvalerate), (PHB), microspheres of this polymer, loaded with flurbiprofen, were successfully prepared by an $\mathrm{O} / \mathrm{W}$ emulsion solvent evaporation method.
A response surface method was implemented to understand the effect of polymer concentration and stabilizer concentration in the microspheres final properties. Results showed that encapsulation efficiency was significantly affected by the two investigated factors, with PVA concentration having a highly negative effect, probably due to flurbiprofen's solubility enhancement in the aqueous phase in the presence of higher amounts of stabilizer. Microspheres mean particle size and size distribution were also affected by the two investigated factors: increasing polymer concentration in the organic phase led to higher mean particle sizes while the increase of surfactant concentration in the continuous phase narrowed the width of particle size distribution. The time for the release of $50 \%$ of the encapsulated drug, $t_{50 \%}$, was not significantly influenced by any of the investigated factors. The in vitro flurbiprofen release profiles from PHBV microspheres were very similar for all the prepared formulations, with more than $90 \%$ of the encapsulated drug released in $\sim 8 \mathrm{~h}$. Results indicated that drug release was essentially controlled by the drug dissolution rate rather than by the hydrolytic degradation of polymeric chains or by the drug diffusion throughout the polymeric matrix. The observed higher dissolution rate of flurbiprofen from PHBV microspheres, when compared to the dissolution rate profile of the pure drug, can be attributed to the dispersion of the amorphous drug in the porous matrix and surface of the microspheres.

These results demonstrate that it is possible to control the quantity of drug loaded in the microspheres and the characteristics of the particle size distribution by changing the quantities of the two investigated factors. On the other hand, the in vitro release profiles of the flurbiprofen-loaded PHB microspheres were not affected by these two factors. For this reason, other factors, in particular the drug-to-polymer ratio, should be investigated in order to control the characteristics of the in vitro drug release profile of the prepared drug delivery system.

\section{Acknowledgements}

Patrícia Coimbra is grateful to Instituto de Investigação Interdisciplinar da Universidade de Coimbra, for PhD Grant III/BIO/35/2005.

\section{References}

Baran ET, Ozer N, Hasirci V. 2002. Poly(hydroxybutyrate-cohydroxyvalerate) nanocapsules as enzyme carriers for cancer therapy: an in vitro study. J Microencapsulation 19:363-376.

Bolourtchian N, Karimi K, Aboofazeli R. 2005. Preparation and characterization of ibuprofen microspheres. J Microencapsulation 22:529-538. 
Chen J, Davis SS. 2002. The release of diazepam from poly(hydroxybutyrate-co-hydroxyvalerate) microspheres. J Microencapsulation 19:191-201.

Cryer B, Feldman M. 1998. Cyclooxygenase-1 and cyclooxygenase-2 selectivity of widely used nonsteroidal anti-inflammatory drugs. Am J Med 104:413-421.

Dubernet C. 1995. Thermoanalysis of microspheres. Thermochim Acta 248:259-269.

Freiberg S, Zhu XX. 2004. Polymer microspheres for controlled drug release. Int J Pharm 282:1-18.

Freitas S, Merkle HP, Gander B. 2005. Microencapsulation by solvent extraction/evaporation: Reviewing the state of the art of microsphere preparation process technology. J Control Rel 102:313-332.

Fu X, Ping Q, Gao Y. 2005. Effects of formulation factors on encapsulation efficiency and release behaviour in vitro of huperzine A-PLGA microspheres. J Microencapsulation 22:705-714.

Gunaratne LM, Shanks RA. 2005. Multiple melting behaviour of poly(3-hydroxybutyrate-co-hydroxyvalerate) using step-scan DSC. Eur Polym J 41:2980-2988.

Hyon SH. 2000. Biodegradable poly(lactic acid) microspheres for drug delivery system. Yonsei Med J 41:720-734.

Khang G, Kim SW, Cho JC, Rhee JM, Yoon SC, Lee HB. 2001. Preparation and characterization of poly(hydroxybutyrateco-hydroxyvalerate) microspheres for the sustained release of 5-fluorouracil. Bio-Med Mater Eng 11:89-105.

Lai MK, Tsiang RCC. 2004. Encapsulating acetaminophen into poly(L-lactide) microcapsules by solvent evaporation technique in $\mathrm{O} / \mathrm{W}$ emulsion. J Microencapsulation 21:307-316.

Li P, Zhao L. 2003. Solubilization of flurbiprofen in $\mathrm{pH}$-surfactant solutions. J Pharm Sci 92:951-956.

Lu Y, Zhang G, Sun D, Zhong Y. 2007. Preparation and evaluation of biodegradable gelatin micro-spheres for intra-articular administration. J Microencapsulation 4:515-524.

Martin MA, Miguens FC, Rieumont J, Sanchez R. 2000. Tailoring of the external and internal morphology of poly-3-hydroxybutyrate microparticles. Colloids Surf B: Biointerf 17:111-116.
Montgomery DC. 2001. Design and analysis of experiments. 5th ed. New York: Wiley.

O’Donnell P, McGinity J. 1997. Preparation of microspheres by the solvent evaporation technique. Adv Drug Del Rev 28:25-42.

Park KM, Kim CK. 1999. Preparation and evaluation of flurbiprofen-loaded microemulsion for parenteral delivery. Int J Pharm 181:173-179.

Park SH, Choi HK. 2006. The effects of surfactants on the dissolution profiles of poorly water-soluble acidic drugs. Int $\mathrm{J}$ Pharm 321:35-41.

Pignatello R, Bucolo C, Spedalieri G, Maltese A, Puglisi G. 2002. Flurbiprofen-loaded acrylate polymer nanosuspensions for ophthalmic application. Biomaterials 23:3247-3255.

Pouton CW, Akhtar S. 1996. Biosynthetic polyhydroxyalkanoates and their potential in drug delivery. Adv Drug Deliv Rev 18:133-162.

Qu XH, Wu Q, Zhang KY, Chen GQ. 2006. In vivo studies of poly(3-hydroxybutyrate-co-3-hydroxyhexanoate) based polymers: Biodegradation and tissue reactions. Biomaterials 27:3540-3548.

Samati Y, Yüksel N, Tarimci N. 2006. Preparation and characterization of poly(D,L-lactic-co-glycolic acid) microspheres containing furbiprofen sodium. Drug Deliv 13:105-111.

Vega E, Egea MA, Valls O, Espina M, García ML. 2006 Flurbiprofen loaded biodegradable nanoparticles for ophthalmic administration. J Pharm Sci 95:2393-2405.

Williams SF, Martin DP, Horowitz DM, Peoples OP. 1999. PHA applications: Addressing the price performance issue I. Tissue engineering. Int J Biol Macromol 25:111-121.

Yiyun C, Jiepin Y. 2005. Solubilization of non-steroidal antiinflammatory drugs in the presence of tween series surfactants. Phys Chem Liq 44:249-256.

Zaghloul AA, Vaithiyalingam SR, Faltinek J, Reddy IK, Khan MA 2001. Response surface methodology to obtain naproxen controlled release tablets from its microspheres with Eudragit L100-55. J Microencapsulation 18:651-662. 